during the next scheduled visit (day 7, 14, 21, 28, 35 and 42) until the QTc normalised.

Results A total of 764 ECG were recorded with 398 participants. Different automatic and manual values of QT and QTc are scattered around different medium. Comparisons of different values of QT: $p=0.1245$ and QTc: $p<0.001$ showed statistically significant differences and the concordance between automatic and manual tests was (QT: Rho_c=0.77 and QTc: Rho_c=0.46). Conclusions Our results indicate no perfect match between automatic and manual methods for QT and QTc. Manual reading remains important to correct any machine errors during clinical studies.

\section{PA-033 PLASMODIUM FALCIPARUM INFECTION IN FEBRILE CONGOLESE CHILDREN: PREVALENCE OF CLINICAL MALARIA TEN YEARS AFTER INTRODUCTION OF ARTEMISININ-COMBINATION THERAPIES}

Mandingha Kosso Etoka-Beka, ${ }^{1}$ Francine Ntoumi, ${ }^{1}$ Michael Kombo, ${ }^{1}$ Julia Deibert, Pierre Poulain, ${ }^{1}$ Christevy Vouvoungui, ${ }^{1}$ Simon Kobawila, ${ }^{3}$

Félix Koukouikila-Koussounda'. ' ${ }^{1}$ FCRM, Republic of Congo; ${ }^{2}$ ITM Tübingen,

Germany; ${ }^{3}$ Université Marien Ngouabi, Republic of Congo

\subsection{6/bmjgh-2016-000260.69}

Background The detailed assessment of the QT and corrected QT interval prolongation is recommended when testing new drugs. Generally the electrocardiograph automatically displays reliable values of the QT interval and corrected QT but morphological variations of the $\mathrm{T}$ wave may cause reading errors; hence the use of manual measurement as an alternative method. Our objective was to evaluate the correlation between the automatic and manual measurement of QT values.

Methods In Kolle from March 2012 to December 2015, an open randomised, phase III-b/IV study comparing dihydroartemisininepiperaquine and pyronaridine-artesunate artemether-lumefantrine was conducted. An electrocardiograph cartridge with 12 electrodes coupled to a computer with the Tele Touch software was used for the electrocardiogram on Day 0 before the study drugs administration and on Day 2, two to four hours after the administration of the last dose of the antimalarial. The manual measurement of QT and QTc was made using the Bazett method [QTcB $\mathrm{m}=($ Number leaded $\times 0.04 \times \mathrm{QTCF})$ / QTcB]. For prolonged QTc cases on Day 2, another measurement was done 